\title{
Searching Sub-Millisecond Pulsars in Accreting Neutron Stars
}

\author{
Alessandro Patruno* \\ Astronomical Institute “A. Pannekoek”, University of Amsterdam, 1098XH Science Park 904, \\ Amsterdam, The Netherlands \\ E-mail: a.patruno@uva.nl
}

\begin{abstract}
Measuring the spin of Accreting Neutron Stars is important because it can provide constraints on the Equation of State of ultra-dense matter. Particularly crutial to our physical understanding is the discovery of sub-millisecond pulsars, because this will immediately rule out many proposed models for the ground state of dense matter. So far, it has been impossible to accomplish this because, for still unknown reasons, only a small amount of Accreting Neutron Stars exhibit coherent pulsations. An intriguing explanation for the lack of pulsations is that they form only on neutron stars accreting with a very low average mass accretion rate. I have searched pulsations in the faintest persistent X-ray source known to date and I found no evidence for pulsations. The implications for accretion theory are very stringent, clearly showing that our understanding of the pulse formation process is not complete. I discuss which sources are optimal to continue the search of sub-ms pulsars and which are the new constraints that theoretical models need to explain to provide a complete description of these systems.
\end{abstract}

High Time Resolution Astrophysics IV - The Era of Extremely Large Telescopes - HTRA-IV,

May 5-7, 2010

Agios Nikolaos, Crete, Greece

* Speaker. 


\section{Introduction}

Neutron stars are objects of great astrophysical importance because the density in their cores can reach values several times higher than normal nuclear matter. At such ultra-high densities the behaviour of matter is not well known, and exotic nuclear processes may take place along with the formation of new states of matter [1, 2]. The properties of ultra-dense matter are determined by the equation of state $(\mathrm{EoS})$ of neutron star matter [3]. This EoS is relatively well known at values below the saturation density of normal nuclear matter, but is extremely uncertain above this threshold. The possibility of constraining the EoS of ultra-dense matter can be achieved only by studying neutron stars and is fundamental for our comprehension of nuclear and sub-nuclear interactions.

Neutron stars exist as a wide variety of objects that can be studied in different electromagnetic wavelengths, ranging from radio to gamma rays. A particularly interesting way to constrain the EoS is via the search of neutron stars spinning at sub millisecond periods. The reason for this is that neutron stars cannot have arbitrarily short spin periods, because the centrifugal force would overcome the effective gravity and induce mass shedding. The minimum achievable spin period of a neutron star depends on its particular EoS because repulsive nuclear interactions have a fundamental role in partially balancing the gravitational pull. It is also possible that self-bound stars exist, like strange stars, who are kept together by the strong interaction and whose rotational period can reach a much smaller value than hadronic neutron stars [2]. Therefore by determining the minimum rotational period it is possible to exclude a large amount of EoS and understand for example the degree of stiffness of ultra-dense matter (see Fig. 1). To date, the fastest known neutron star is the radio pulsar PSR J1748-2446ad, spinning at $716 \mathrm{~Hz}$ ( $\sim 1.4 \mathrm{~ms}$ [ [†). Although this period corresponds to a rotational velocity of $\sim 15 \%$ the speed of light (assuming a neutron star radius of 10 $\mathrm{km}$ ), it is still compatible with basically all realistic EoS. Radio pulsars with spin periods beyond the $1.4 \mathrm{~ms} \mathrm{limit}$ are difficult to detect due to observational limitations like the unknown dispersion measure. In X-rays instead the search for pulsation is not biased at least down to $0.5 \mathrm{~ms}$ and in this sense the subclass of accreting neutron star is the optimal target for sub-ms pulsar searches.

The most interesting accreting neutron stars are those in which the companion star has a low mass: they are called neutron star low mass X-ray binaries (NS-LMXBs). These systems are very old (with ages of the order of $10^{8}-10^{9} \mathrm{yr}$ ) and the neutron star has spent a substantial fraction of its life accreting gas and spinning up via transfer of angular momentum [5]. This means that there is high chance of finding a millisecond pulsar, and possibly a sub-ms pulsar, in a NS-LMXB. In this work I present the first results of a deep pulse search in these kind of X-ray binaries.

\section{Accreting Neutron Stars}

The number of known NS-LMXBs in our galaxy is $\sim 100$ (see for example [7]). There are also many binaries in which the companion is a high mass star, but they are of little interest here because their relatively young age and the intense magnetic field of the neutron star prevent them to having been spun up to the millisecond range [8].

In NS-LMXBs instead, the majority of neutron stars is old, and their magnetic field is believed to have decayed to values around $10^{8}-10^{9} \mathrm{G}$. Some gas leaves the companion via Roche Lobe 


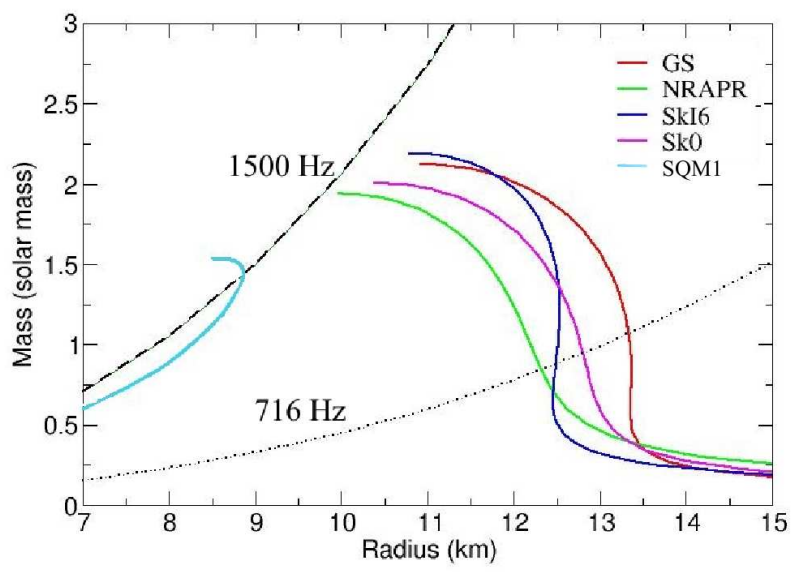

Figure 1: Mass-Radius relation for neutron stars with different Equation of State (see [3] for a description of the EoS used). The dotted and dashed lines show the constraints that a neutron star spinning at $1500 \mathrm{~Hz}$ and $716 \mathrm{~Hz}$ would imply on the EoS: any point below these lines cannot represent the Mass and Radius of the spinning neutron star, since the centrifugal forces would imply mass shedding and disruption of the object.

Overflow and forms an accretion disc around the neutron star. When the gas loses enough angular momentum and arrives at the innermost regions of the disc, two phenomena can take place:

- if the neutron star has an external magnetic field larger than $\sim 10^{7}-10^{8} \mathrm{G}$, the gas is (partially) channeled towards the magnetic poles

- if the neutron star has no relevant external magnetic field, then the gas keeps spiraling down to the innermost stable circular orbit (ISCO) and then plunges on the neutron star surface. If the ISCO is below the neutron star surface, as it is the case for some EoS, then the disc extends down to the neutron star surface.

In the case of channeled accretion, the gas might flow in the magnetosphere in ordered structures producing two sites of intense $\mathrm{X}$-ray radiation at the magnetic poles. If the magnetic and rotational axes are misaligned, the X-rays are modulated at the spin period of the neutron star and are called "accreting X-ray pulsars". In the second scenario the accreting gas does not produce any sufficient emission asymmetry and no pulsations are produced.

\section{Accreting Millisecond X-ray Pulsars}

Among the $\sim 100$ NS-LMXBs, only a small number show X-ray pulsations. A very small sub-group is composed by NS-LMXBs with very long spin periods (tens to hundreds of seconds, see [8]), while 13 systems show X-ray pulsations in the millisecond range (Accreting Millisecond X-ray Pulsars, AMXPs, see Table 1).

All Accreting Millisecond X-ray pulsars share similar characteristics: 
Table 1: Accreting Millisecond X-ray Pulsars

\begin{tabular}{|c|c|c|c|c|c|c|c|}
\hline Source & $\begin{array}{c}v_{s} \\
(\mathrm{~Hz})\end{array}$ & $\begin{array}{c}P_{\text {orb }} \\
(\mathrm{min})\end{array}$ & $\begin{array}{c}f_{x} \\
\left(M_{\odot}\right)\end{array}$ & $\begin{array}{c}M_{c, \min } \\
\left(M_{\odot}\right)\end{array}$ & Type I Bursts & B-O & Reference \\
\hline SAX J1808.4-3658. & 401 & 121 & $3.8 \times 10^{-5}$ & 0.043 & Yes & Yes & \\
\hline XTE J1751-305 . . . & 435 & 42.4 & $1.3 \times 10^{-6}$ & 0.014 & No & No & \\
\hline XTE J0929-314 . . . . & 185 & 43.6 & $2.9 \times 10^{-7}$ & 0.0083 & No & No & 4110 \\
\hline XTE J807-294 . . . . & 190 & 40.1 & $1.5 \times 10^{-7}$ & 0.0066 & No & No & (4) \\
\hline XTE J1814-338 . . . & 314 & 257 & $2.0 \times 10^{-3}$ & 0.17 & Yes & Yes & \\
\hline IGR J00291+5934 . . & 599 & 147 & $2.8 \times 10^{-5}$ & 0.039 & No & No & \\
\hline HETE J1900.1-2455 & 377 & 83.3 & $2.0 \times 10^{-6}$ & 0.016 & Yes & Yes & 15 \\
\hline Swift J1756.9-2508 & 182 & 54.7 & $1.6 \times 10^{-7}$ & 0.007 & No & No & 6 \\
\hline Aql $X-1 \ldots \ldots \ldots$ & 550 & 1194 & N/A & N/A & Yes & Yes & \\
\hline SAX J1748.9-2021. & 442 & 522 & $4.8 \times 10^{-4}$ & 0.1 & Yes & No & \\
\hline NGC6440 X-2 . . . . & 206 & 57 & $1.6 \times 10^{-7}$ & 0.0067 & No & No & \\
\hline IGR J17511-3057 .. & 245 & 208 & $1.1 \times 10^{-3}$ & 0.13 & Yes & Yes & (2) \\
\hline Swift J1749.4-2807. & 518 & 529 & $5.5 \times 10^{-2}$ & 0.59 & No & No & [21] \\
\hline
\end{tabular}

$v_{s}$ is the spin frequency, $P_{\text {orb }}$ the orbital period, $f_{X}$ is the X-ray mass function, $M_{c, \min }$ is the minimum companion mass. The 6 th and 7 th columns identify bursting sources and those that show Burst-Oscillations (B-O). The last column shows references for the timing parameters. The references chosen are those in which the most precise timing parameters are reported.

- pulsations are sinusoidal, with little or no harmonic content

- the companion star has a low mass from $1 M_{\odot}$ down to $0.007 M_{\odot}$

- the binary orbital period is short, between 0.7 and $20 \mathrm{hr}$.

The rotation of the neutron star modulates the X-ray emission and gives a sinusoidal shape for the pulses, mainly because of a projected area effect. The fact that all AMXPs have a low mass companion star is a consequence of binary evolution: if the companion star were massive, then accretion would take place either via an intense wind or via a dynamical/thermal timescale that would rapidly reduce the mass of the companion and possibly engulf the whole system. The mass transfer becomes stable when the mass ratio between the donor and the neutron star becomes close or smaller than $\sim 1$. Once a stable mass transfer sets in, the neutron star is spun up on timescales of $10^{7}-10^{8} \mathrm{yr}$, which are rather short when compared to the lifetime of the binary ( $\sim 10^{9} \mathrm{yr}$, see [5]).

To have a sufficiently intense mass transfer from a low mass companion it is necessary to have Roche Lobe Overflow, because the stellar wind is too weak to provide enough gas for the production of intense X-ray pulses. Since the companion stars have usually a mass much smaller than $1 M_{\odot}$, reaching the impressively small value of $\sim 7$ Jupiter masses (see Table 1 ), they are usually compact or ultra-compact systems: to overflow the Roche Lobe it is necessary that the companion is very close to the neutron star [5].

\section{Why Does Only a Small Number of Accreting Neutron Stars Pulsate ?}

A consequence of standard accretion theory and of the recycling scenario [6] is that all NSLMXBs that are accreting at a sufficiently high rate must show X-ray pulsations. However, as anticipated in the previous sections, only 13 out of $\sim 100$ NS-LMXBs do pulsate. The reason for this is still unknown. Many models exist today that try to provide an answer to this problem, but 
none has been considered conclusive yet. Some models suggest that the dipolar magnetic field is buried in the neutron star crust [22], thus preventing any channeled accretion. Other models predict a smearing of pulsations due to gravitational lensing [23], or to scattering in a hot corona surrounding the pulsar [24] or to alignment between the spin and the magnetic axes [25]. Other models have proposed the onset of magneto-hydrodynamical instabilities, that would develop at the magnetosphere-disc boundary, like Rayleigh-Taylor instabilities that destroy the coherence of the accretion [26]. It is beyond the scope of this contribution to explain such models, but what is important to stress once more is that none of them has found a final confirmation yet.

A major breakthrough in our comprehension of the pulse formation process was achieved with the discovery of intermittency in some AMXPs. The first reported episode of intermittency was done for the AMXP HETE J1900.1-2455 [15]. This pulsar showed approximately 2 months of continuous pulsations during an outburst that started in 2005. The pulsations then disappeared and were not detected for the following 5 years, up to the time of writing this contribution. Two other intermittent sources were then discovered (Aql X-1 and SAX J1748.9-2021, see Table 1). The most remarkable was Aql X-1: a single $120 \mathrm{~s}$ long episode of pulsations was detected with high significance among more than 1.2 Ms of observations. SAX J1748.9-2021 showed instead repeated episodes of intermittency with pulsations appearing and disappearing on timescales of a few hundred seconds and with a possible connection with the occurrence of Type I X-ray bursts (see Table 1 and references therein). These three sources are of great importance because they might bridge the gap between AMXPs and non-pulsating NS-LMXBs. If the mechanism that produces intermittency is also responsible for the lack of pulsations in many NS-LMXBs, then any pulse formation model must be able to explain the existence of rapid timescales as short as $\sim 100 \mathrm{~s}$ for the formation and destruction of X-ray pulses.

\section{Nuclear Powered X-ray Pulsars}

The gas accreted on the neutron star surface accumulates and might give rise to thermonuclear explosions if specific temperature and density conditions are met. Thermonuclear explosions (a.k.a. Type I X-ray bursts) are sudden releases of nuclear energy that happen on timescales of a few tens to hundreds of seconds. Although the energy released per accreted baryon $(\sim 200 \mathrm{MeV} / \mathrm{baryon})$ is much larger than the nuclear energy released during stable burning ( $\sim 5 \mathrm{MeV} /$ baryon), the unstable nuclear reactions taking place during Type I X-ray burst release energy that completely dominates the production of X-ray radiation. During Type I X-ray bursts, nuclear powered oscillations at (or very close to) the spin frequency of the neutron star are sometimes observed [27].

The precise mechanism for the formation of burst oscillations is still unknown and current models propose a relation with global modes of oscillation of the neutron star surface, thermonuclear hurricanes or hot-spot emission asymmetries due to the thermonuclear flame spreading (see for example [28] and references therein).

We know 11 NS-LMXBs that show burst oscillations, and they are of high importance because 6 of these have never shown accretion powered pulses; therefore their spin would have never been known. Considering nuclear powered and accretion powered oscillations, we have a sample of 23 NS-LMXBs with known confirmed spin periods, which means that now we begin having a good sample for accreting neutron star spin distribution. In a recent paper [14], I reported the updated 
spin frequency distribution of the 23 known accretion and nuclear powered millisecond X-ray pulsars. The distribution shows still a sharp cutoff around $730 \mathrm{~Hz}$ with $99 \%$ confidence level and with median value for the spin frequency of $415 \mathrm{~Hz}$. The spin distribution cutoff limit of $730 \mathrm{~Hz}$ still resists after about 7 years and with a sample size doubled from its first calculation [29]. The only claimed sub-ms pulsar is the $1122 \mathrm{~Hz}$ accreting neutron star XTE J1739-285 [30]. However, this result is still unconfirmed.

Since all persistent AMXPs have shown a rather low average mass accretion rate, it is well possible that the key propriety that determines why these sources show pulsations while other NS-LMXBs do not is indeed the low average mass accretion rate. It has been proposed that the dipolar magnetic field of neutron stars is buried in the crust during accretion [22]. If the Ohmic diffusion timescale, that regulates the diffusion of the magnetic field in the plasma, is shorter than the accretion timescale, then the magnetic filed is able to spread outside the neutron star surface and affect the accretion process in the disc. The buried magnetic field model is one of the possibilities to explain the lack of pulsations in the majority of NS-LMXBs. However, a considerable theoretical effort needs to be still made, because the current model rely on several approximations that might considerably affect the results. A simple prediction of this model, which seems to be at the origin of its success, is that the magnetic field is buried when the mass accretion rate is typically above $2 \%$ of the Eddington limit.

It seems that all AMXPs have a low long-term average mass accretion rate [31], which is much smaller than that of non pulsating NS-LMXBs, suggesting that this is the right direction to go when searching for pulsators among NS-LMXBs. However, some care has to be taken when calculating the long term average mass accretion rate of AMXPs, since the distance and the recurrence time of many of them is rather uncertain.

To verify the consistency of the buried magnetic field model it is therefore crucial to search for pulsations in those NS-LMXBs that have a very low average mass accretion rate. Recently, a new sub-class of LMXBs has been discovered, called Very Faint X-ray Sources (VFXSs) whose main property is to have very faint peak luminosities always below $10^{36} \mathrm{erg} \mathrm{s}^{-1}$ [32]. If the accretor is a neutron star, then these are the optimal targets for any pulse search campaign.

\section{The Very Faint X-ray Source 1RXS J171824.2-402934}

The faintest persistent VFXS known to date is 1RXSJ171824.2-402934. The source shows Type I X-ray bursts with photospheric radius expansion. Assuming a nearly Eddington limited emission during the luminosity peak, a distance of $6.5 \pm 0.5 \mathrm{kpc}$ was inferred [33]. A Chandra observation carried in 2004 [34] has shown a persistent emission with a total unabsorbed $0.5-10 \mathrm{keV}$ flux of $(9.7 \pm 1.7) \times 10^{-12} \mathrm{erg} \mathrm{cm}^{-2} \mathrm{~s}^{-1}$ which corresponds to a luminosity of $4.9 \times 10^{34} \mathrm{erg} \mathrm{s}^{-1}$, for a distance of $6.5 \mathrm{kpc}$. The X-ray luminosity is approaching $10^{-4} L_{E d d}$ and the inferred mean mass transfer rate is $\sim 9 \times 10^{-12} \mathrm{M}_{\odot} \mathrm{yr}^{-1}$, both are in perfect agreement with the requirement set by the buried magnetic field model to produce pulsations. If the neutron star atmosphere is strongly contaminated by helium material, the distance obtained from the Type I burst should be increased up to $9 \mathrm{kpc}$. Even in this case the luminosity is very faint $\left(9.4 \times 10^{34} \mathrm{erg} \mathrm{s}^{-1}\right)$ and the considerations remain unchanged. The spectrum of the source has been fitted with an absorbed power law with a 

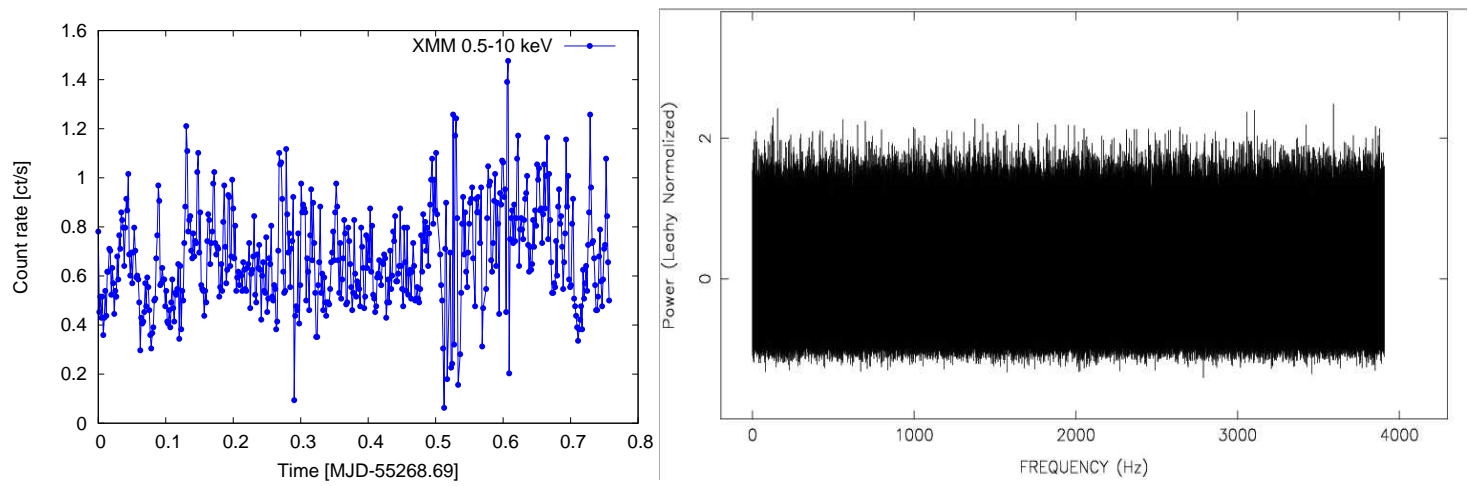

Figure 2: X-ray count-rate (left panel) and power spectrum (right panel) of the Very Faint Source 1RXSJ171824.2-402934. The data refer to an XMM-Newton observation of $65 \mathrm{ks}$ long data in the 0.5$10 \mathrm{keV}$ energy band. No significant $\mathrm{X}$-ray flux variations are observed, and a simple power spectrum shows no relevant features.

photon index $\Gamma=2.09_{-0.24}^{+0.22}$ and a column density $N_{H}=1.32_{-0.12}^{+0.16} \times 10^{22} \mathrm{~cm}^{-2}$ which seems to be typical of NS-LMXBs.

In a recent work [35], it was proposed that this source has the unique characteristic of being the shortest ultra-compact binary known to date: its orbital period should be less than 7 min, compared with the 11 min of the X-ray binary $4 \mathrm{U} 1820-30$. The determination of the period is very uncertain, and has to be taken with caution, but it is interesting anyway to see that the discovery of pulsations would allow not only a test of magnetic field evolution theories and the determination of the neutron star spin but also the confirmation of the shortest orbital period known. I obtained a $65 \mathrm{ks}$ long data from a pointed XMM-Newton observation of this source, taken with the EPIC-pn camera set up in timing mode to allow a search for pulsations. The observed source flux is indeed very low, and never goes above $1.5 \mathrm{ct} / \mathrm{s}$ in the $0.5-10 \mathrm{keV}$ energy band. This can be translated in an observed average mass accretion rate of about $6 \times 10^{-12} \mathrm{M}_{\odot} \mathrm{yr}^{-1}$ (which is $0.02 \%$ Eddington) for an assumed distance of $6.5 \mathrm{kpc}$ and using spectral parameters as reported in [34]. The lightcurve and the power spectrum of the XMM observation are reported in Figure 2.

An inspection of the power spectrum of the lightcurve shows no evident pulsations at frequencies below $2 \mathrm{kHz}$. Given the background and source count rates, if a signal with an rms amplitude larger than $2 \%$ were present, then a power spectrum would show a spike with a signal-to-noise ratio of at least 5. However, this search technique makes the non-trivial assumption that the signal stays in one single Fourier frequency bin during the whole observation. If the suggestion of a short period made in [35] is correct, then the power spectrum is heavily affected by Doppler shifts due to the orbital motion of the neutron star around the companion and that would spread the signal in a large number of Fourier frequency bins.

To take this possibility into account I performed a deep pulse search by using the sidebandsearch technique explained by [36] and [37]. This search technique is optimal when the length of the time series is much larger than the orbital period, because a family of sidebands in the Fourier power spectrum will appear around the pulsar spin frequency. An important requirement to increase the sensitivity is that many orbital periods are included in the observation so to create 
a large number of sidebands. The result of the search did not return any significant spin frequency candidate. Although the calculation of proper upper limits is strongly dependent on the assumed orbital and spin parameters during the search, preliminary limits of less than $1 \% \mathrm{rms}$ on the pulse amplitude can be placed (see [38]). Acceleration searches and calculations of more stringent upper limits are currently under way.

Upper limits of $1 \% \mathrm{rms}$ are already very constraining for all theories of pulse formation, and they question the validity of the argument that a low value of average mass accretion rate favours the appearance of pulsations. It remains to be investigated whether the persistence of 1RXSJ171824.2402934, and therefore the continuous accretion of mass, might have a role on the pulse formation process. Therefore it would be interesting to search for pulsations also in VFXSs which are transients instead of persistent.

\section{Theoretical implications}

The lack of pulsations in the VFXS 1RXSJ171824.2-402934 has several theoretical implications. First, it shows that a pulse search program to unveil the existence of sub-ms pulsars in NS-LMXBs is more difficult than predicted. The existence of sub-ms pulsars has not been demonstrated so far and deep X-ray data searches have not returned positive results (see for example [39] for a deep search in the NS-LMXB 4U 1820-30). In a recent paper [14], I discussed how the only two AMXPs for which a long term spin period evolution is constrained (SAX J1808.4-3658 and IGR J00291+5934) do not show any evidence for a short-timescale spin evolution. The AMXP SAX J1808.4-3658 shows a long term spin-down over an observed baseline of 10 years, while IGR J00291+5934 shows the spin increasing at a rate that would bring it in the sub-ms range in $\sim 10$ Gyr. If this is a common behaviour among NS-LMXBs, it might imply that none of the NS-LMXBs have been spun up for a sufficiently long time to reach sub-ms periods. The lack of sub-ms pulsars might therefore be a simple consequence of binary evolution (see [40] and references therein). To establish whether sub-ms pulsars exist or not, it might be interesting therefore to determine the long term evolution of other AMXPs, although this might be prohibitive given the long recurrence time between outbursts of several of them. A possible strategy to overcome this problem would be to increase the sample of known AMXPs via a deep search of targeted candidates, like the VFXS, to determine whether the effect of a low mass accretion rate is indeed an important parameter for the pulse formation.

A second consequence of the study of 1RXSJ171824.2-402934 is that a low average mass accretion rate is not sufficient to guarantee the formation of X-ray pulses by itself. Recently, the buried magnetic field model was further developed [41] with encouraging results: the external dipole magnetic field can be reduced on timescales of months and the amount by which the magnetic field in the neutron star ocean changes depends on the average mass accretion rate and also on the total amount of mass accreted. This would in principle explain why 1RXSJ171824.2-402934 does not show pulsations: if the amount of mass accreted is sufficiently large, then pulsations might not form. 


\section{Acknowledgments}

I would like to thank R. Wijnands and N. Degenaar for several stimulating discussions on the Very Faint Source properties, A. L. Watts. and Y. Cavecchi for discussions on the Equation of State of ultra-dense matter and thermonuclear burst models.

\section{References}

[1] Weber, F., Negreiros, R., Rosenfield, P., \& Torres I Cuadrat, A. 2007, Quark Confinement and the Hadron Spectrum VII, 892, 515

[2] N. K. Glendenning, Compact Stars, Nuclear Physics, Particle Physics, and General Relativity, 2nd ed. (Springer-Verlag, New York, 2000).

[3] Lattimer, J. M., \& Prakash, M. 2001, The Astrophys. J. 550, 426

[4] Hessels, J. W. T., Ransom, S. M., Stairs, I. H., Freire, P. C. C., Kaspi, V. M., \& Camilo, F. 2006, Science, 311, 1901

[5] Tauris, T. M., \& van den Heuvel, E. P. J. 2006, Compact stellar X-ray sources, 623 , (Cambridge University Press, 2006).

[6] Alpar, M. A., Cheng, A. F., Ruderman, M. A., \& Shaham, J. 1982, Nature, 300, 728

[7] Liu, Q. Z., van Paradijs, J., \& van den Heuvel, E. P. J. 2007, Astronomy and Astrophysics, 469, 807

[8] Bildsten, L., et al. 1997, The Astrophys. J. Supp., 113, 367

[9] Hartman, J. M., Patruno, A., Chakrabarty, D., Markwardt, C. B., Morgan, E. H., van der Klis, M., \& Wijnands, R. 2009, The Astrophys. J., 702, 1673

[10] Papitto, A., Menna, M. T., Burderi, L., di Salvo, T., \& Riggio, A. 2008, Month. Not. of the Royal Astron. Soc., 383, 411

[11] Galloway, D. K., Chakrabarty, D., Morgan, E. H., \& Remillard, R. A. 2002, The Astrophys. J. Letters , 576, L137

[12] Patruno, A., Hartman, J. M., Wijnands, R., Chakrabarty, D., \& van der Klis, M. 2010a, The Astrophys. J. 717, 1253

[13] Papitto, A., di Salvo, T., Burderi, L., Menna, M. T., Lavagetto, G., \& Riggio, A. 2007, Month. Not. of the Royal Astron. Soc., 375, 971

[14] Patruno, A. 2010, arXiv:1006.0815

[15] Galloway, D. K., Morgan, E. H., Krauss, M. I., Kaaret, P., \& Chakrabarty, D. 2007,The Astrophys. J. Letters , 654, L73

[16] Patruno, A., Altamirano, D., \& Messenger, C. 2010b, Month. Not. of the Royal Astron. Soc., 403, 1426

[17] Casella, P., Altamirano, D., Patruno, A., Wijnands, R., \& van der Klis, M. 2008, The Astrophys. J. Letters, 674, L41

[18] Patruno, A., Altamirano, D., Hessels, J. W. T., Casella, P., Wijnands, R., \& van der Klis, M. 2009, The Astrophys. J. , 690, 1856

[19] Altamirano, D., et al. 2010a, The Astrophys. J. Letters, 712, L58 
[20] Papitto, A., Riggio, A., Di Salvo, T., Burderi, L., D’Aì, A., Iaria, R., Bozzo, E., \& Menna, M. T. 2010, arXiv: 1005.4827

[21] Altamirano, D., et al. 2010b, arXiv:1005.3527

[22] Cumming, A., Zweibel, E., \& Bildsten, L. 2001, The Astrophys. J., 557, 958

[23] Wood, K. S., Ftaclas, C., \& Kearney, M. 1988, The Astrophys. J. Letters, 324, L63

[24] Titarchuk, L., Cui, W., \& Wood, K. 2002, The Astrophys. J. Letters,, 576, L49

[25] Lamb, F. K., Boutloukos, S., Van Wassenhove, S., Chamberlain, R. T., Lo, K. H., \& Miller, M. C. 2009, The Astrophys. J. Letters, 705, L36

[26] Kulkarni, A. K., \& Romanova, M. M. 2008, Month. Not. of the Royal Astron. Soc., 386, 673

[27] Strohmayer, T., \& Bildsten, L. 2006, Compact stellar X-ray sources, 113, (Cambridge University Press, 2006).

[28] Watts, A. L. 2008, A DECADE OF ACCRETING MILLISECOND X-RAY PULSARS, American Institute of Physics Conference Series, 1068, 199

[29] Chakrabarty, D., Morgan, E. H., Muno, M. P., Galloway, D. K., Wijnands, R., van der Klis, M., \& Markwardt, C. B. 2003, Narute, 424, 42

[30] Kaaret, P., et al. 2007, The Astrophys. J. Letters, 657, L97

[31] Heinke, C. O., Jonker, P. G., Wijnands, R., Deloye, C. J., \& Taam, R. E. 2009, The Astrophys. J. , 691, 1035

[32] Wijnands, R. 2006, Populations of High Energy Sources in Galaxies, 230, 328, Cambridge University Press, 2006.

[33] Kaptein, R. G., in’t Zand, J. J. M., Kuulkers, E., Verbunt, F., Heise, J., \& Cornelisse, R. 2000, , Astronomy and Astrophysics, 358, L71

[34] in’t Zand, J. J. M., Cornelisse, R., \& Méndez, M. 2005, Astronomy and Astrophysics, 440, 287

[35] in't Zand, J. J. M., Jonker, P. G., Bassa, C. G., Markwardt, C. B., \& Levine, A. M. 2009, Astronomy and Astrophysics, 506, 857

[36] Jouteux, S., Ramachandran, R., Stappers, B. W., Jonker, P. G., \& van der Klis, M. 2002, Astronomy and Astrophysics, 384, 532

[37] Ransom, S. M., Cordes, J. M., \& Eikenberry, S. S. 2003, The Astrophys. J., 589, 911

[38] Vaughan, B. A., et al. 1994, The Astrophys. J., 435, 362

[39] Dib, R., Ransom, S. M., Ray, P. S., Kaspi, V. M., \& Archibald, A. M. 2005, The Astrophys. J, 626, 333

[40] Deloye, C. J. 2008, 40 Years of Pulsars: Millisecond Pulsars, Magnetars and More, AIP Conference Proceedings, 983, 501

[41] Cumming, A. 2008, A DECADE OF ACCRETING MILLISECOND X-RAY PULSARS, American Institute of Physics Conference Series, 1068, 152 